\title{
LOCALLY TRIVIAL HOMOTOPY FUNCTORS AND THEIR DOMAIN OF REPRESENTATION
}

\author{
MICHAEL D. ALDER
}

(Received 5 December 1974)

\begin{abstract}
The well known theorem of E. H. Brown on the representation of Homotopy Functors is extended to a larger domain category. This is accomplished by requiring the functor to satisfy a further condition, that of being locally trivial: the condition is both necessary and sufficient for the extension and is satisfied by functors arising from locally trivial fibre bundles such as $K$-theory.
\end{abstract}

\section{Introduction}

1.1. Notation. $\mathscr{H} t p_{*}$ denotes the category of spaces with distinguished (base) point and homotopy classes of continuous point-preserving maps. $\mathscr{H} \mathscr{C}_{*}$ denotes the subcategory having objects those spaces possessing the homotopy type of a $\mathrm{C}$. W. complex, and $\mathscr{S}_{*}$ the category of pointed sets and point preserving maps.

Let

$$
\mathscr{H}: \mathscr{T o p}_{*} \rightarrow \mathscr{H t p}{ }_{*}
$$

denote the obvious projection functor and $[f]$ the homotopy class of the map $f$.

1.2 Preamble. Suppose $\Gamma: \mathscr{H} t p_{*} \rightarrow \mathscr{S}_{*}$ is a (contravariant) homotopy functor in the sense of Brown (1965). Then by Brown's theorem there is a complex $\mathrm{K}$ in $\mathscr{H C}_{*}$ such that

$$
\Gamma \mid \mathscr{H} \mathscr{C}_{*} \text { is naturally equivalent to }[-, K]_{*} \mid \mathscr{K} \mathscr{C}_{*}
$$

and we say $\Gamma$ is representable, represented by $K$ on $\mathscr{H}_{*} \mathscr{C}_{*}$.

In this paper I show that with an extra condition on the functor $\Gamma$, namely that it be locally trivial in a sense to be defined, $\Gamma$ is represented by $K$ on a larger category that contains, inter alia, the one-point compactifications of complexes. The condition of local triviality is a natural one abstracted from locally trivial fibre-bundle functors such as Grothendieck-Atiyah-Hirzebruch $K$. 
1.3. Remark. Recall some facts and definitions of category theory, Freyd (1964).

A contravariant functor is called cocontinuous if it takes colimits to limits. Any colimit can be expressed in terms of coproducts and coequalisers, or equivalently in terms of coproducts and pushouts, hence a contravariant functor is cocontinuous iff it takes coproducts to products and pushouts to pullbacks.

The contravariant homfunctor is always cocontinuous and the Adjoint Functor theorem Freyd (1964) implies a partial converse: with suitable extra conditions on the domain category, a cocontinuous functor taking values in $\mathscr{S}_{*}$ is naturally equivalent to a homfunctor. This is close to Brown's theorem Brown (1965). However neither $\mathscr{H}_{*}$ nor $\mathscr{H}_{*}$ in general have colimits (Eckmann and Hilton (1963/64)). The condition of being a homotopy functor makes sense on $\mathscr{H}_{*} p_{*}$ and amounts to the following: that the functor takes coproducts to products and (in place of taking pushouts to pullbacks) that it takes the reduced mapping torus (Dold (1966)) to a "weak pullback". Since this sufficies to obtain representability on $\mathscr{K}_{\mathscr{C}_{*}}$, it follows that it implies cocontinuity, i.e. that the functor takes what colimits it finds in $\mathscr{H C} \mathscr{C}_{*}$ to limits in $\mathscr{S}_{*}$.

The homotopy functors referred to in this paper will be supposed then to satisfy the extra condition of taking pushouts to pullbacks (hence coequalisers to equalisers) when the former occur. This condition is superfluous on $\mathscr{H} \mathscr{C}_{*}$ and clearly necessary on any category.

\section{Locally Trivial Functors}

2.1. Remark. Bundle functors, such as Grothendieck-Atiyah-Hirzebruch $K$-theory, assign to a suitable space some equivalence class of the set of bundles of given fibre over that space. This set may inherit some extra structure from the fibre, but there is always a distinguished bundle, viz. the product or trivial bundle. One is concerned usually with locally trivial fibre bundles, that is to say, one admits a bundle provided it is accompanied by a cover of the (base) space by open sets such that the restriction of the bundle to each open set is a trivial bundle.

We abstract this idea in the following definition:

2.2. Definition. Let $\Gamma: \mathscr{H}_{*} \rightarrow \mathscr{S}_{*}$ be a homotopy functor. We say that $\Gamma \circ \mathscr{H}$ is locally trivial on $X \in \mathscr{T}_{o p_{*}}$ iff $\forall x \in \Gamma \circ \mathscr{H}(X)$, there is a cover of $X$ by open sets $\left\{U_{j}: j \in J\right\}$, with inclusions $\left\{i_{j}: U_{j} \rightarrow X\right\}_{J}$ such that $\forall j \in J,(\Gamma \circ \mathscr{H}) \times$ $\left.i_{j}(x)\right)={ }^{*}$ where ${ }^{*}$ is the distinguished element in $\Gamma \circ \mathscr{H}\left(U_{j}\right)$.

If $\Gamma \circ \mathscr{H}$ is locally trivial on $X$ for each $X$ in a subcategory $\mathcal{M}$ of $\mathscr{T}_{o p_{*}}$, we say that $\Gamma \circ \mathscr{H}$, or by abuse of language $\Gamma$, is locally trivial on $\mathcal{M}$ (or $\mathscr{H} \mathcal{M}$ ). 
2.3. REMARK. It is easy to see that this abuse of language is innocuous. If $X$ and $Y$ have the same homotopy type and $\Gamma \circ \mathscr{H}$ is locally trivial on $X$, then it is locally trivial on $Y$ also. In fact, it suffices that $X$ dominate $Y$.

2.4. Remark. If $K$ is a locally contractible space in the sense of admitting an open cover with null-homotopic inclusions, then the homfunctor

$$
[-, K]_{*}: \mathscr{H}_{t} p_{*} \rightarrow \mathscr{S}_{*} \text { is locally trivial, }
$$

where the distinguished element of $[X, K]_{*}$ is the homotopy class of the constant map from $X$ to ${ }^{*} \in K$.

In particular if $K$ has the homotopy type of a C. W. complex it is locally contractible in this sense (Spanier (1966)) and hence $[-, K]_{*}$ is locally trivial. It follows that if we want $\Gamma$ to be represented by a complex, then we need $\Gamma$ locally trivial.

\section{Paracomplexes}

3.1. REMARK. In this section I define a category of spaces, the paracom. plexes, and give some examples. Broadly speaking, a paracomplex is 'almost' a complex except that it may have local pathologies of a non contractible sort; in 4 I show that locally trivial functors ignore such pathologies, and hence obtain the extension.

I shall suppose certain well known properties of partitions of unity and nerves of open covers: these may be found in Spanier (1966). In particular the following notation is convenient:

If $\left\{f_{i}: X \rightarrow[0,1], j \in J\right\}$ is a partition of unity on $X$, and if $\forall j \in J, U_{j}=$ $f_{j}^{-1}(0,1]$ is the associated cover, then $N_{J}$ will denote the nerve of this cover and $\pi_{J}: X \rightarrow N_{J}$ is the canonical map sending each $x \in X$ to its various barycentric co-ordinates, the $f_{i}(x)$.

A set of partitions of unity on a space $X$ is cofinal if for every partition of unity on $X$, there is an element of the set which refines it.

\subsection{Definition. A topological space $X$ is paracomplex iff}

1. it is paracompact and path connected and

2. it admits a cofinal set of partitions of unity such that for each element $\left\{f_{i}\right\}$, of this set, the diagram

$$
\coprod_{J} U_{j} \stackrel{[\Sigma]}{\longrightarrow} X \stackrel{\mid(\pi,)}{\rightarrow} N_{J}
$$

is a cokernel in $\mathscr{H} t p$, where $\forall j \in J, U_{j}=f_{i}^{-1}(0,1]$, and $\Sigma \mid U_{j}$ is the inclusion. 
3.3. REMARK. It is easy to see that

$$
\coprod_{J} U_{i} \stackrel{[\Sigma]}{\longrightarrow} X \stackrel{[\pi,]}{\longrightarrow} N_{J}
$$

a cokernel in $\mathscr{H} t p$ implies that

$$
\coprod_{J} U_{i}^{\prime} \stackrel{\left[\Sigma^{\prime}\right]}{\rightarrow} X \stackrel{\left[\pi_{J}\right]}{\rightarrow} N_{J}
$$

is a cokernel in $\mathscr{H}_{*}$, where ${ }^{*} \in X$ is any point,

$$
* \in N_{J}=\pi_{J}(*) \text {, }
$$

and $\forall j \in J, U_{j}^{\prime}=U_{j} \cup[0,1]$ where 0 is identified to any point of $U_{j}$ and 1 is *, the distinguished point.

Moreover if $U_{j}^{\prime}$ has 0 identified to the point $p \in U_{i}$ then $\left(U_{j}^{\prime},{ }^{*}\right)$ and $\left(U_{j}, p\right)$ have the same homotopy type in $\mathscr{H} t p_{*}$, where ${ }^{*}$ and $p$ are the respective distinguished points.

In short, by such contrivances as pinning 'tails' on to our covering sets we can avoid being obsessive about base points.

3.4. Remark. It is easy to see that any connected simplicial complex is a paracomplex with $\left\{U_{j}\right\}_{J}$ the star open cover or refinements of it and $\pi_{J}$ a homeomorphism. Further, if $X$ is locally contractible in the sense that it admits an open cover with null homotopic inclusions (and is also path connected and paracompact), then it is a paracomplex iff it has the homotopy type of a complex. For we may choose a null homotopic cover and a subordinate partition of unity; then the identity map $1: X \rightarrow X$ is the cokernel of $[\Sigma]$, whence $X$ and $N_{J}$ are of the same homotopy type.

It is easy to verify that the "Hawaian Ear-ring", the space obtained by factoring out a Cauchy sequence in $S^{\prime}$ is a paracomplex that is not a complex. This generalises:

3.5. Proposition. Let $X$ be a path connected paracompact space and $A \subset X$ a discrete subspace such that for a cofinal set of neighbourhoods $\left\{V_{\lambda}: \lambda \in \Lambda\right\}$ of $A, X-V_{\lambda}$ is a simplicial complex.

Then $X$ is a paracomplex.

Proof. It simplifies the argument to suppose $A$ a single point: let $V_{\lambda}$ and $V_{\mu}$ be neighbourhoods of $A$ with $\bar{V}_{\mu} \subset V_{\lambda}$ and $X-V_{\mu}$ a simplicial complex, $K$.

We construct a partition of unity on $X$ as follows:

Let $f_{A}$ be a continuous map from $\bar{V}_{\lambda}$ to $[0,1]$ that is 1 on $\bar{V}_{\mu}$ and 0 on $\partial V_{\lambda}$. Since $X$ is paracompact, hence normal, this can always be done.

For $x \in X-V_{\mu}$, (i.e. $\left.x \in K\right)$ put $f_{j}(x)=\left(1-f_{A}(x)\right) g_{j}(x)$, where the $g_{j}$ are the barycentric co-ordinates of $x \in K$. It is clear that this set of $f_{i}$ together with $f_{A}$ gives a partition of unity on $X$. 
Moreover, the nerve of the associated covering is a complex $\hat{K}$ comprising $K$ together with an extra vertex $\hat{A}$ and the corresponding simplices. The diagram:

$$
\coprod_{J} U_{i} \stackrel{[\Sigma]}{\rightarrow} X \stackrel{[\pi,]}{\longrightarrow} \hat{K}
$$

has $\left\{U_{j}\right\}_{J}$ the star open cover of $K$ together with the open set on $A$ containing $\bar{V}_{\mu}$ and contained in $V_{\lambda}, f_{A}^{-1}(0,1)$. Call it $V_{\lambda}$ still.

This diagram is a cokernel (as is the diagram arising from refinements of the $U_{j}$ ). For certainly

$$
\forall j \in J, \quad\left[\pi_{J} \mid U_{i}\right]=*,
$$

sinee $\pi_{s} \mid U_{i}$ has image in the star of the corresponding vertex which is contractible. And if

$$
\pi^{\prime}: X \rightarrow M \text { has }\left[\pi^{\prime}\right] \circ[\Sigma]=* \text {, }
$$

(i.e. $\pi^{\prime} \mid U_{j}$ null homotopic, $\forall_{j}$ with $\pi^{\prime} \mid V_{\lambda}$ null homotopic) then since $\pi \mid X-$ $V_{\wedge}$ is the identity we may define

$$
\alpha: \hat{K} \rightarrow M
$$

by $\alpha=\pi^{\prime} \hat{K}$-(star $\hat{A}$ ), and on star $\hat{A}$ we may define $\alpha$ to be the homotopy which contracts $\pi^{\prime} \mid V_{\lambda}$ to the constant map.

(More formally if $x \in \operatorname{star} A$ has barycentric co-ordinates $f_{1}(x), \cdots, f_{n}(x)$ and there is a homotopy

$$
\begin{array}{lll}
H: V_{\lambda} \times I \rightarrow M & \text { with } H \mid V_{\lambda} \times 0=\pi^{\prime} \\
& \text { and } H \mid V_{\lambda} \times 1=* \text {, the }
\end{array}
$$

the constant map to some point of $M$, put

$$
\begin{aligned}
\alpha(x) & =\alpha\left(f_{1}(x), f_{2}(x), \cdots, f_{n}(x), f_{A}(x)\right) \\
& =H\left(\left(g_{1}(x), g_{2}(x), \cdots, g_{n}(x)\right), f_{A}(x)\right)
\end{aligned}
$$

for $x \in X_{\lambda}-V_{\mu}$, and $\alpha(x)=* \forall x \in V_{\mu}$.

Because

$$
\left.\begin{array}{lll}
f_{A}=0 & \text { on } & \partial V_{\lambda} \\
f_{A}=1 & \text { on } & V_{\mu}
\end{array}\right\}, \alpha \text { is continuous. }
$$

Clearly $\left[\alpha \circ \pi_{J}\right]=\left[\pi^{\prime}\right]$ and $\alpha$ is unique up to homotopy. The result follows.

3.6. COROLlaRY. The one point compactification of a simplicial complex is a paracomplex. 


\section{The Representation Theorem Extended}

4.1. Proposition. A locally trivial Homotopy functor $\Gamma: \mathscr{H}_{t} p_{*} \rightarrow \mathscr{S}_{*}$ which is represented by a complex $K$ on $\mathscr{H C}_{*}$ is also represented by the same complex on the subcategory of $\mathscr{H}_{*}$ containing those spaces having the homotopy type of a paracomplex.

Proof. Take an $x \in \Gamma X$, for $X$ a paracomplex, and a partition of unity $\left\{f_{i}: j \in J\right\}$ on $X$ such that

$$
\coprod_{J}\left(f_{i}^{-1}(0,1]\right) \stackrel{|\Sigma|}{\longrightarrow} X \stackrel{[\pi,]}{\longrightarrow} N_{J}
$$

is a cokernel in $\mathscr{H} t p$, and $\Gamma \Sigma(x)=*$.

By the remark 3.3. we obtain the corresponding diagram in $\mathscr{H}_{*}$.

To show $\Gamma$ is representable on $X$, if $K$ is the classifying space for $\Gamma \mid \mathscr{H C}_{*}$, it suffices to show that there is a unique map $\alpha: X \rightarrow K$ such that $\Gamma \alpha(k)=x$, where $k \in \Gamma K$ corresponds to the identity under the natural equivalence between $[K, K]_{*}$ and $\Gamma K$.

For this will establish that the map

$$
\begin{aligned}
\tilde{k}:[X, K]_{*} & \rightarrow \Gamma X \\
g & \sim \Gamma g(k)
\end{aligned}
$$

is bijective. Since $\tilde{k}$ is manifestly a natural transformation the result will follow.

Now by Brown's theorem $K$ is a complex, and by cocontinuity of $\Gamma$ the diagram

$$
\Gamma N_{\jmath} \stackrel{\Gamma[\pi, 1}{\longrightarrow} \Gamma X \stackrel{\Gamma[\Sigma]}{\longrightarrow} \Gamma \coprod_{j} f_{i}^{-1}(0,1]
$$

is a kernel in $\mathscr{S}_{*}$. In particular it is exact and so there is an $n \in \Gamma N_{J}$ such that $\Gamma\left[\pi_{J}\right](n)=x$.

Now there is certainly a unique map in $\mathscr{H}_{*} f: N_{J} \rightarrow K$ such that $\Gamma f(k)=n$, since $\Gamma$ is represented by $K$ on $\mathscr{H}_{C_{*}}$ and $N_{J}$ is a complex. Composing with $\left[\pi_{J}\right]$ gives the required map from $X$ to $K$.

It remains to show $f \circ\left[\pi_{J}\right]$ is unique.

Suppose that $g, h: X \rightarrow K$ are two maps such that

$$
\Gamma[g](k)=\Gamma[h](k)=x .
$$

We may choose a partition of unity $\left\{V_{l}: l \in L\right\}$ on $X$ such that

$$
\forall l \in L, \quad g \mid V_{1} \text { and } h \mid V_{1} \text { are null homotopic. }
$$

Then $[g]$ and $[h]$ both factor through $N_{L}$ by $[\tilde{g}]$ and $[\tilde{h}]: N_{L} \rightarrow K$ respectively. 
But

$$
\coprod_{L} V_{l} \stackrel{\left[\Sigma_{L}\right]}{\longrightarrow} X \stackrel{\left[\pi_{L}\right]}{\longrightarrow} N_{L}
$$

is a cokernel, so $\Gamma\left[\pi_{L}\right]$ is mono and $\Gamma[\tilde{g}](k)=\Gamma[\bar{h}](k)=n^{\prime} \in \Gamma N_{L}$ such that $\Gamma\left[\pi_{L}\right]\left(n^{\prime}\right)=x \in \Gamma X$.

But there is precisely one map, say $\beta$, from $N_{L}$ to $K$ with the property that $\Gamma \beta(k)=n^{\prime}$.

Hence $[\tilde{g}]=[\tilde{h}],[g]=[h]$, and the result follows.

4.2. CoROllary. Locally trivial, cocontinuous homotopy functors from $\mathscr{H}_{*}$ to $\mathscr{S}_{*}$ are representable on the one point compactifications of simplicial complexes.

I am grateful to Professor C. T. C. Wall for drawing this and related problems to my attention.

\section{References}

E. H. Brown (1965), 'Abstract Homotopy Theory', Trans. Am. Math. Soc. 119, 79-85.

P. Freyd (1964), 'Abelian Categories', Harper and Row.

E. Spanier (1966), 'Algebraic Topology', McGraw-Hill.

B. Eckmann and P. Hilton (1963/64), 'Unions and Intersections in Homotopy Theory', Comm. Math. Helv. 38, 293-307.

A. Dold (1963), 'Partitions of Unity in the theory of Fibrations', Ann. Math. 78, 223-255.

A. Dold (1966), 'Halbexakte Homotopiefunktoren', Springer Lecture Note Series No. 12.

Department of Mathematics

University of Western Australia

Nedlands, W. A. 6009. 